LA W REN CE LIVERMORE N A TIONAL LABORATORY

Proton Radiography of Magnetic Fields in a Laser Produced Plasma

S. Le Pape, P. Patel, S. Chen, R. Town, D. Hey, A. Mackinnon

July 6,2010

High Energy Density Physics 
This document was prepared as an account of work sponsored by an agency of the United States government. Neither the United States government nor Lawrence Livermore National Security, LLC, nor any of their employees makes any warranty, expressed or implied, or assumes any legal liability or responsibility for the accuracy, completeness, or usefulness of any information, apparatus, product, or process disclosed, or represents that its use would not infringe privately owned rights. Reference herein to any specific commercial product, process, or service by trade name, trademark, manufacturer, or otherwise does not necessarily constitute or imply its endorsement, recommendation, or favoring by the United States government or Lawrence Livermore National Security, LLC. The views and opinions of authors expressed herein do not necessarily state or reflect those of the United States government or Lawrence Livermore National Security, LLC, and shall not be used for advertising or product endorsement purposes. 


\title{
Proton radiography of magnetic fields in a laser produced plasma
}

\author{
Sebastien Le Pape ${ }^{*}$, Pravesh Patel, Sophia Chen, Richard Town, Dan Hey, Andrew \\ Mackinnon \\ Lawrence Livermore National Laboratory, Livermore, CA 94550
}

\begin{abstract}
Magnetic and electric fields generated by the interaction of long-pulse laser beams with plasmas relevant to inertial confinement fusion have been measured. A proton beam generated by the interaction of an ultraintense laser with a thin metallic foil is used to probe laser-produced fields. The proton beam is temporally short (on the order of a picosecond) and highly laminar, and hence equivalent to a virtual point source which makes it an ideal source for point projection radiography. We have investigated, using face-on and side-on radiography, fields due to the non-colinearity of temperature and density gradients at laser intensities around $5 \times 10^{13} \mathrm{~W} / \mathrm{cm}^{2}$.
\end{abstract}

\section{Introduction}

The generation of and electric fields in a laser plasma interaction is of great interest in the field of high energy density physics $\left[{ }^{1}\right]$. Megagauss B fields generated in the laser irradiation of a solid target can modify heat flow and alter electron density, $\mathrm{n}_{\mathrm{e}}$, and electron temperature, $\mathrm{T}_{\mathrm{e}}$, distributions, leading to plasma instabilities $\left[{ }^{2}\right]$. In the case of long pulse (nanosecond scale) and low intensity irradiation $\left(10^{13} \mathrm{~W} / \mathrm{cm}^{2}\right)$, the dominant mechanism for the generation of B fields is the non-collinear density and temperature gradient, $\left(\nabla \mathrm{n}_{\mathrm{e}} \times \nabla \mathrm{T}_{\mathrm{e}}\right)\left[{ }^{3}\right],\left[{ }^{4}\right]$. These fields are generated at the edge of the expanding plasma bubble, the shape of which is related to the size of the focal spot. The Faraday rotation equation combined with a simplified version of Ohm's law can be used to describe the B field evolution $\left[{ }^{5}\right]$ :

\footnotetext{
* Permanent address: 7000 east avenue 94550 livermore, CA,USA, lepape2@1lnl.gov, phone 925-4226201, fax 925-422-2253
} 
$\partial B / \partial t \approx \nabla \times(v \times B)-\frac{1}{e n_{e}} \nabla n_{e} \times \nabla T_{e}[1]$

where $\mathrm{v}$ is the plasma velocity.

Previous experiments have studied B fields in ICF relevant plasmas. The first experiments to measure B fields in laser plasmas used Faraday rotation of an optical probe beam $\left[{ }^{6}\right]$. This technique is limited to densities much lower than critical density and thus cannot be used to access fields close to the critical density, where the optical probe beam is strongly deflected by the density gradient. Some recent experiments have used proton deflectometry $\left[^{7}\right]$ to measure B fields in long pulse plasmas. In ref [6] monoenergetic protons are generated as fusion products from the reaction $\mathrm{D}+3 \mathrm{He}->\alpha+\mathrm{p}$ with an associated $150 \mathrm{ps}$ time resolution and $50 \mu \mathrm{m}$ spatial resolution. In the experiment discussed here, protons are generated from the interaction of a short pulse laser $(0.7 \mathrm{ps})$ with a thin metal foil. The proton source is then polychromatic (from a few hundred $\mathrm{keV}$ to about $40 \mathrm{MeV}$ ) $\left[{ }^{8}\right]$ and emitted during $\sim 1$ ps.

\section{Experimental arrangement}

The experiment was carried out at the Titan laser facility at LLNL. A short pulse laser beam (80J in $700 \mathrm{fs}$ ) is focused by an $\mathrm{f} / 3$ off-axis parabola to a $10 \mu \mathrm{m}$ spot leading to a peak intensity of approximately $10^{19}$ $\mathrm{W} / \mathrm{cm}^{2}$. A $50 \mu \mathrm{m}$ thick tungsten foil is used to produce the proton beam $\left[{ }^{9}\right],\left[{ }^{10}\right]$. At these intensities the proton energy spectrum extends with an exponential slope that cuts off at $30 \mathrm{MeV}$. A 1ns long pulse beam at $\lambda=1064 \mathrm{~nm}$ is focused using a phase plate to a $1 \mathrm{~mm}$ spot with an intensity of $5 \times 10^{13} \mathrm{~W} / \mathrm{cm}^{2}$ on a $5 \mu \mathrm{m}$ thick Aluminum target (figure 1).

The proton beam probes the B field in a face-on geometry (normal to the target surface). Proton deflectometry $\left[{ }^{11}\right]$ is used to estimate the amplitude and the spatial distribution of the fields. A Copper mesh, either 400 or 1000 lines per inch (LPI), is placed between the proton target and the long pulse target. The proton detector is $6 \mathrm{~cm}$ from the proton target; it is composed of a multilayer assembly of radiochromic film $\left.{ }^{12}\right]$. Because the ions release most of their energy at the Bragg peak, each layer of film provides a quasi-monochromatic image. The spatial resolution is 55 microns with a 400 LPI mesh and 30 microns with a 1000 LPI mesh.

\section{Experimental results}

Given a flat-top focal spot intensity distribution radiation-hydrodynamic simulations predict an axially symmetric azimuthal B-field and the absence of radial E-fields in the expanding plasma $\left[{ }^{13}\right]$. This geometry 
implies that protons probing the plasma face-on are only sensitive to B fields while side-on deflectometry is only sensitive to E fields $\left[{ }^{14}\right]$. The B fields are probed face-one with different time delays between the long pulse and the short pulse; the time delays take into account the time of flight of the protons from the proton target to the probed target. Figure 2 shows the mesh deflections in the proton beam at different time delays of 200, 400, 900 and 1100 ps. A straight mesh is used as a reference to estimate the mesh displacement In fig 2.a. a proton pile-up is visible at the edge of the plasma bubble where the B fields are the strongest. At longer delays (900 ps and $1.1 \mathrm{~ns}$ ), the proton pile-up becomes asymmetrical, probably due to the presence of resistive instabilities $\left[{ }^{15}\right]$. At later time the fluid behaviour near the buble edge can be dominated by field and resistive effects, the plasma ratio of thermal to field energy cam become smaller than 1 which gives the rise to the possibility of resistive instabilities.

Figure 3 shows the B field profile as a function of time modeled with the 2D LASNEX [13] hydrocode. The B field package includes the full braginskii cross-field transport model and spontaneously generates fields in the presence of nonparallel $\mathrm{T}_{\mathrm{e}}$ and $\mathrm{n}_{\mathrm{e}}$ gradients. The simulation used the experimental parameters of $420 \mathrm{~J}$ laser energy of $1.053 \mu \mathrm{m}$ light, delivered in a $1 \mathrm{~ns}$ pulse and focused to a $1 \mathrm{~mm}$ diameter spot. It shows that the B field is concentrated in a hemispherical shell surrounding the plasma plume. After an initial increase in the amplitude of the B field, the field is frozen in with its spatial structure evolving as the plasma expands.

Assuming that the protons only probe B fields in the face-on geometry, the mesh distortions in the detector plane are directly related to the product $\mathrm{B} \times \mathrm{dl}$ where $\mathrm{B}$ is the amplitude of the $\mathrm{B}$ field and $\mathrm{dl}$ is the spatial size of the B field along the line of sight. A routine was developed to track the mesh distortions induced by the B fields. We use the part of the image where the mesh is straight as a reference to calculate the mesh displacement

The $\mathrm{B}$ field can be estimated from the mesh distortion $\delta$, taking into account the geometry of the experiment (i.e distance from the mesh to the detector), from the relation $\mathrm{B} \approx \delta . \mathrm{E}_{\mathrm{p}} 0.5 \cdot \mathrm{dl}^{-1}$, where $\mathrm{E}_{\mathrm{p}}$ is the proton energy and $\mathrm{dl}$ is the scale length of the plasma. As in ref [6], $\mathrm{dl}$ is is estimated to be constant over a plasma layer where the field are located. LASNEX predicts a plasma layer of about 50-150 $\mu \mathrm{m}$. The main source of error is to estimate the distance over which the B fields are present, the amplitude of the B field vary linearly with this value. 
Figure 4 shows the B field profile at 400 ps deduced from (a) the experimental data, and (b) the profile calculated with LASNEX. Both profiles exhibit the same annular shape. The experimental profile shows a maximum field strength of $0.4 \mathrm{MG} \pm 50 \%$ while the simulated profile shows a maximum strength field of 0.45 MG. The field structure deduced from the proton deflectometry data is similar to the field structured simulated with LASNEX.

\section{Conclusion}

Proton deflectometry has been applied to study the fields induced by the interaction of a long pulse laser with a thin metallic target. The characteristics of the laser generated proton beam (short pulse duration $\approx$ 1ps, high energy up to $30 \mathrm{Mev}$ ) provide high-resolution deflectograms of the field. At intensities around $5 \times 10^{13} \mathrm{~W} / \mathrm{cm}^{2}$, the amplitude of the fields is approximately 0.4 MGauss. The experimental results have been compared to $2 \mathrm{D}$ hydro simulations and are in good overall agreement.

This work performed under the auspices of the U.S. Department of Energy by Lawrence Livermore National Laboratory under Contract DE-AC52-07NA27344. 
[1] S. Eliezer, The Interaction of High-Power Lasers with Plasmas (IOP Publishing, Bristol, 2002).

[2] S. H. Glenzer, Contrib. Plasma Phys. 40, 36 (2000)

[3] J. A. Stamper and B.H.Ripin., Phys. Rev. Lett. 34, 000138 (1971)

[4] M. G. Haines, Phys. Rev. Lett. 78, 2 (1997)

[5] J. A. Stamper, K. Papadopoulos, R. N. Sudan, E. A. Mclean, and J. M. Dawson Phys. Rev. Lett., 26,1012,(1971)

[6] Borghesi, M.; MacKinnon, A. J.; Gaillard, R.; Willi, O.; Pukhov, A.; Meyer-Ter-Vehn, J., Physical Review Letters, Volume 80, Issue 23, June 8, 1998, pp.5137-5140

[7] Li, C. K.; Séguin, F. H.; Frenje, J. A.; Rygg, J. R.;et al. Physical Review Letters, vol. 97, Issue 13, id. 135003

[8] Clark, E. L.; Krushelnick, K.; Davies, J. R.; Zepf, M.;et al.., Physical Review Letters, Volume 84, Issue 4, January 24, 2000, pp.670-673

[9 ] S.C. Wilks, A.B. Landgon, M. Roth, M. Singh, S. Hatchett, M. H. Key, R. A. Snavely: Phys. Plas. 8, 542 (2001)

[10] S. Krasch, S. Dusterer, H. Schwoerer, F. Ewald, D. Habs, M. Hegelich, G. Preztler, A. Pukhov, K. Witte, R. Sauerbrey, Phys. Rev. Lett. 91. 015001 (2003)

[11] MacKinnon, A. J.; Patel, P. K.; Town, et al., Review of Scientific Instruments, Volume 75, Issue 10, pp. 35313536 (2004).

[12] W. L. C. McLaughlin, et al., Nucl. Instr. Meth. Phys. Res. A, 302, 165 (1991) 
[13] G. B. Zimmerman and W. L. Kruer, Comments Plasma Phys. Control. Fusion 2, 51 (1975).

[14] R. P. J. Town et al., Bull. Am. Phys. Soc. 50, 123 (2005)

[15] Li, C. K.; Séguin, F. H.; Frenje, J. A.; Rygg, et al.,Physical Review Letters, vol. 99, Issue 1, id. 01500

\section{Figure caption}

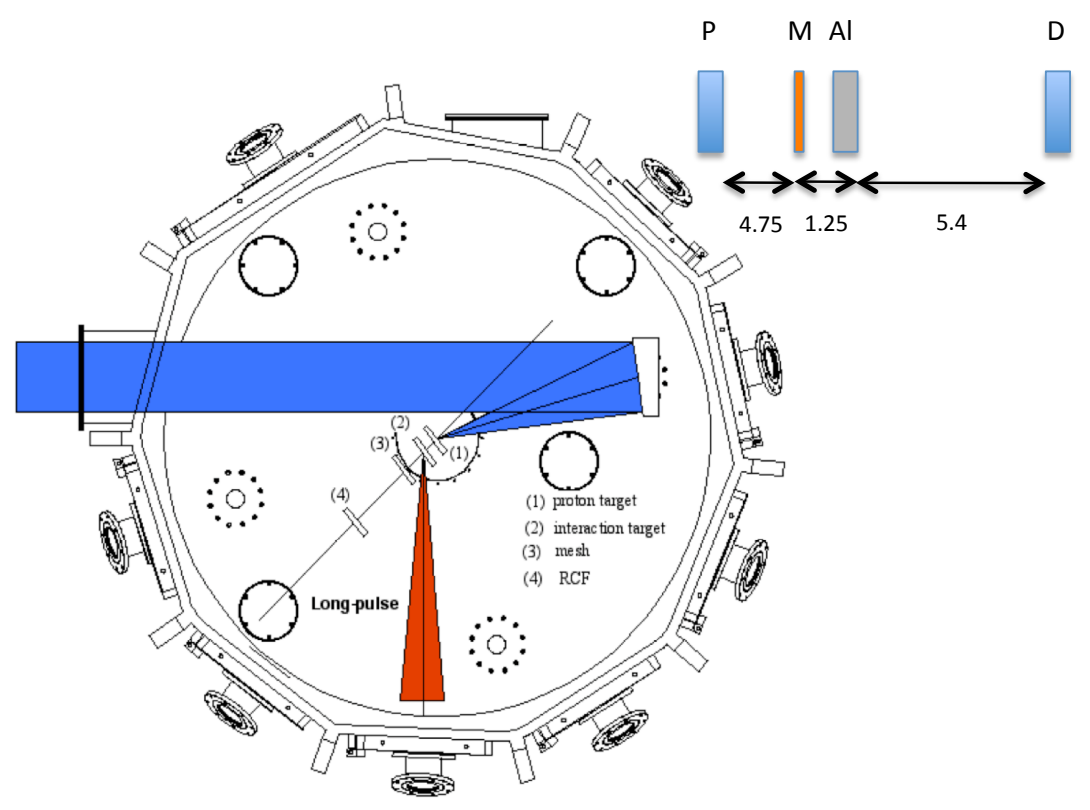

Figure 1: Experimental setup of the proton deflectometry experiment. $\mathrm{P}=$ proton, $\mathrm{M}=\mathrm{mesh}, \mathrm{Al}=$ aluminum, $\mathrm{D}=$ detector. Distances are in $\mathrm{mm}$. 


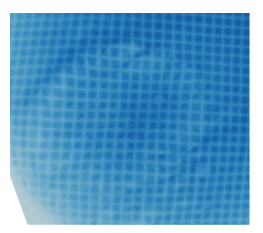

a

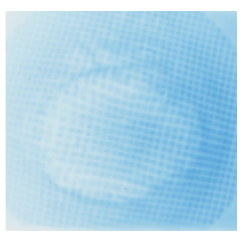

b

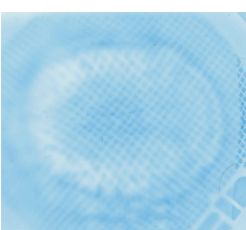

$\mathrm{c}$

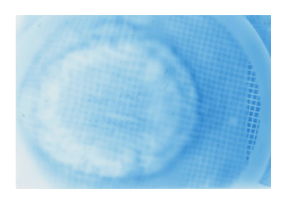

d

Figure 2: Results of face-on deflectometry of $11.3 \mathrm{Mev}$ protons through the long pulse plasma at different times a) $200 \mathrm{ps}$, b) $400 \mathrm{ps} \mathrm{c)} 600 \mathrm{ps}$ and d) $1100 \mathrm{ps}$

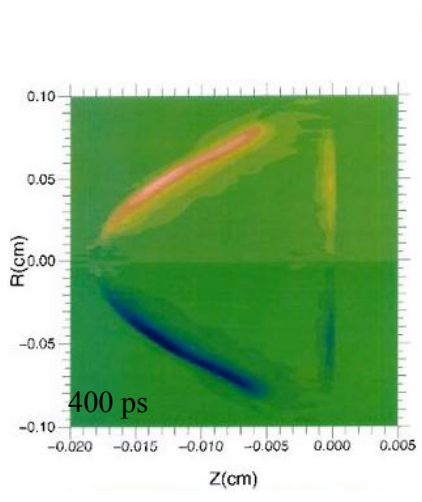

a

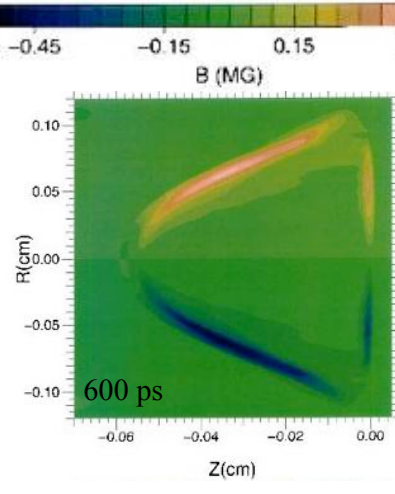

b

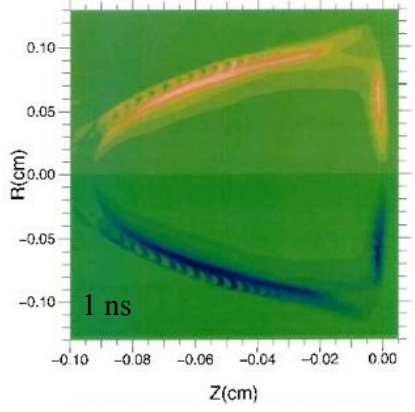

C

Figure 3: LASNEX simulated B field profile at different time delays a) $400 \mathrm{ps}$, b) $600 \mathrm{ps}$, c) $1100 \mathrm{ps}$ 


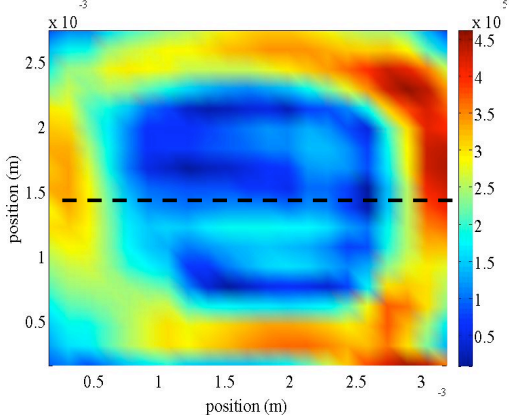

a

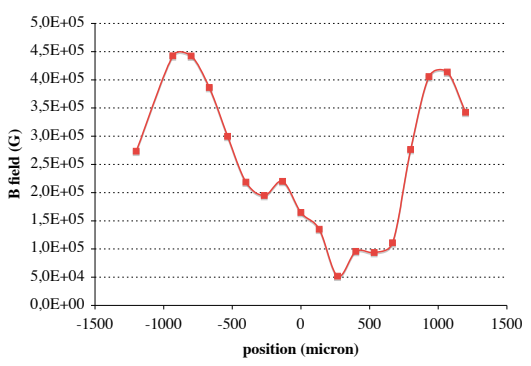

C

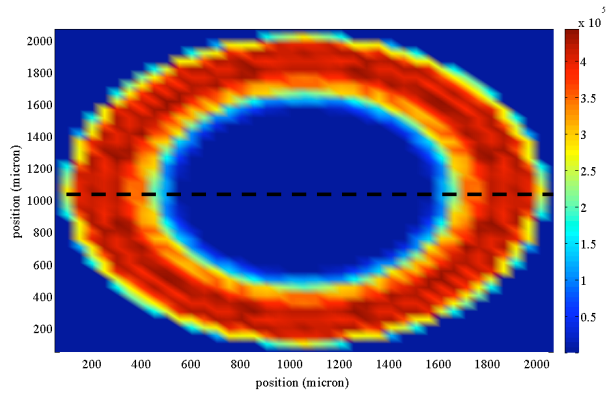

b

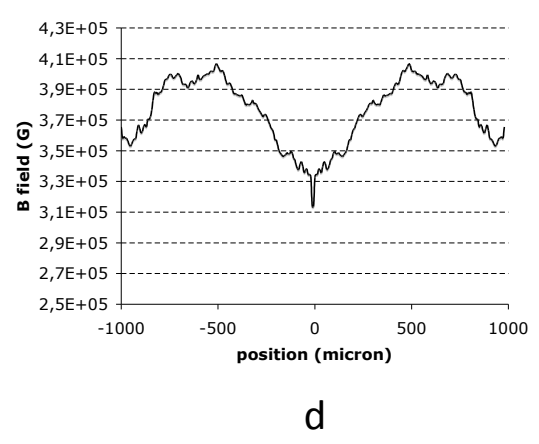

Figure 4: a) face-on B field profile deduced from the deflectograms c) face B field profile deduced from the LASNEX simulation, b) line-out on the experimental B field profile along the axis drawn on the image 4 c). d) line-out on the simulated B field profile along the axis drawn on the image $4 \mathrm{c}$ ). 